\section{Distal Pancreatectomy Combined with Celiac Axis Resection in Treatment of Carcinoma of the Body/Tail of the Pancreas: Proceed with Caution}

\section{TO THE EDITORS:}

We read with interest the paper published by $\mathrm{Wu}$ and colleagues (Ann Surg Oncol. 2010 Mar 3) and congratulate the authors for their work. ${ }^{1}$ However, the present work deserves some comments.

In this trial, all patients underwent contrast-enhanced computed tomography (CT) scan to evaluate surgical resectability. Patients without metastases of other organs, peritoneal dissemination, or any significant comorbidities were assigned to receive radical distal pancreatectomy, but 6 patients $(2.9 \%)$ underwent palliative distal pancreatectomy, and $46(22.3 \%)$ patients' operations were aborted because micrometastases of the liver and peritoneum, diffuse invasion to adjacent tissue, or invasion to major vessels was found during exploratory surgery.

In group $\mathrm{A}, 11$ patients who underwent distal pancreatectomy combined with resection of the celiac axis (CA) had stage III tumor according to the 6th edition of the American Joint Committee on Cancer pancreatic cancer staging system. In group C, 11 patients who did not receive surgical exploration may have stage III or IV tumor. Patients who did not receive surgical exploration may have micrometastases of the liver and peritoneum. So, tumor stage was not similar at baseline in the two study groups. The difference may influence slightly the survival rate.

Patients with unresectable stage III (13\%) and stage IV disease $(55 \%)$ together account for the overwhelming majority of patients who present with newly diagnosed pancreatic cancer and have uniformly poor survival. ${ }^{2}$

Surgical resection of the pancreatic tumor is generally considered to be inappropriate in patients with metastatic disease (stage IV), as the metastases are virtually always multifocal and associated with survival duration of approximately 6 months. ${ }^{3}$

Resection of tumors involving the CA is technically difficult because these vessels are surrounded. Even if a portion of the CA or superior mesenteric artery (SMA) is resected and reconstructed, the majority of patients with such locally advanced disease also have synchronous systemic metastases, even if not apparent on imaging studies. ${ }^{4,5}$

Elect bias is defined as any systematic error resulting in inaccurate estimation of the outcome of a study. Just as the authors stated, this study with retrospective nature may have an inevitable selection bias. Therefore the conclusion that distal pancreatectomy combined with resection of the $\mathrm{CA}$ is a safe and effective procedure for patients with carcinoma of the body/tail of pancreas associated with CA invasion needs to be taken cautiously.

\section{Sun Hu Yang ${ }^{1,2}$, and Ke Feng Dou ${ }^{1}$}

${ }^{1}$ Department of Hepatobiliary Surgery, Fourth Military Medical University, Xijing Hospital, Xi'an, Shaanxi, China; ${ }^{2}$ Department of General Surgery, People's Hospital of Zhangye City, Zhangye City, Gansu, China

e-mail: doukefeng2010@126.com

Published Online: 22 October 2010

(c) Society of Surgical Oncology 2010

\section{REFERENCES}

1. Wu X, Tao R, Lei R, et al. Distal pancreatectomy combined with celiac axis resection in treatment of carcinoma of the body/tail of the pancreas: a single-center experience. Ann Surg Oncol. 2010 Mar 3.

2. Sener SF, Fremgen A, Menck HR, Winchester DP. Pancreatic cancer: a report of treatment and survival trends for 100,313 patients diagnosed from 1985-1995, using the National Cancer Database. J Am Coll Surg. 1999;189:1-7.

3. Katz MH, Hwang R, Fleming JB, Evans DB. Tumor-nodemetastasis staging of pancreatic adenocarcinoma. CA Cancer $J$ Clin. 2008;58:111-25.

4. Nagakawa T, Mori K, Nakano T, et al. Perineural invasion of carcinoma of the pancreas and biliary tract. $\mathrm{Br} J$ Surg. 1993;80:619-621.

5. Kayahara M, Nagakawa T, Konishi I, et al. Clinicopathological study of pancreatic carcinoma with particular reference to the invasion of the extrapancreatic neural plexus. Int J Pancreatol. 1991;10:105-111. 